Musées, Patrimoine et Culture scientifiques et techniques

$162 \mid 2015$

novembre-décembre 2015

\title{
De l'usage des réseaux socio-numériques comme supports d'une médiation culturelle en ligne
}

\section{Sébastien Magro}

\section{OpenEdition \\ Journals}

Édition électronique

URL : http://journals.openedition.org/ocim/1593

DOI : $10.4000 /$ ocim. 1593

ISSN : 2108-646X

Éditeur

OCIM

Édition imprimée

Date de publication : 1 novembre 2015

Pagination : $37-40$

ISSN : 0994-1908

Référence électronique

Sébastien Magro, « De l'usage des réseaux socio-numériques comme supports d'une médiation culturelle en ligne », La Lettre de I'OCIM [En ligne], 162 | 2015, mis en ligne le 01 septembre 2016, consulté le 19 avril 2019. URL : http://journals.openedition.org/ocim/1593 ; DOI : 10.4000/ocim.1593

Ce document a été généré automatiquement le 19 avril 2019

Tous droits réservés 


\section{De l'usage des réseaux socio- numériques comme supports d'une médiation culturelle en ligne}

\section{Sébastien Magro}

Communiquer et discuter sur Facebook au musée des Beaux-Arts d'Angers.

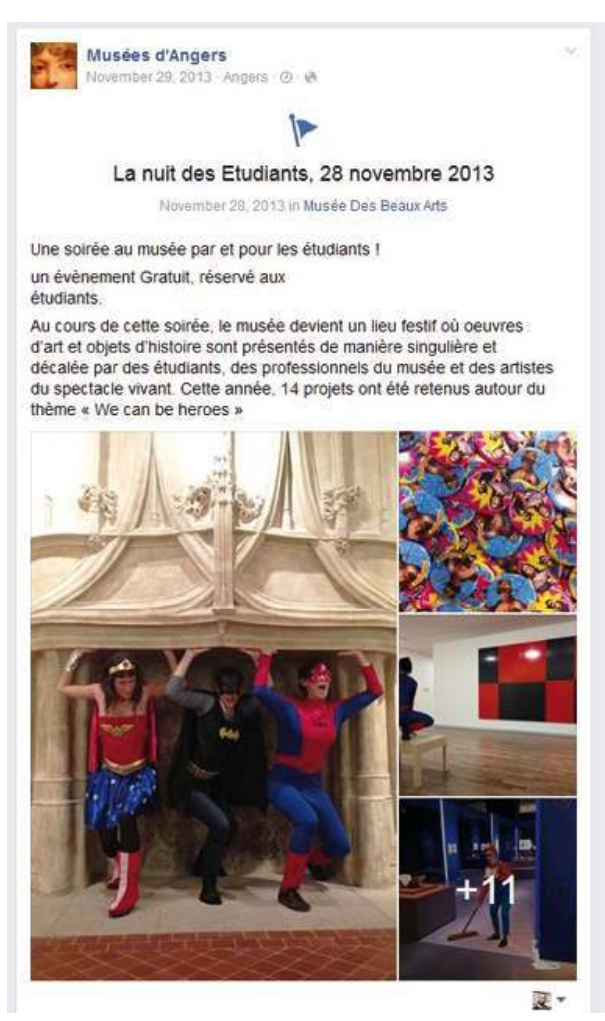

1 Les réseaux socio-numériques (RSN), apparus au milieu des années 2000, ont été progressivement adoptés par les institutions culturelles et patrimoniales francophones depuis 2010. Au-delà des usages communément attendus de ce type de plateformes 
(communication, marketing, informations pratiques), certains établissements y déploient des actions de médiation culturelle en s'appuyant sur les échanges avec leurs abonnés, ainsi que sur ceux qui naissent entre ces publics en ligne.

2 S'inscrivant dans le prolongement des actions menées sur le Web par les musées, les RSN formulent la promesse de l'appropriation des thématiques par les visiteurs, les invitant à participer à la création du propos qui entoure collections et expositions. À partir d'exemples issus du monde francophone, nous proposons quelques pistes de réflexion sur la nature de cette médiation en ligne, abordant les enjeux de l'ouverture des échanges entre musées et institutions, ainsi que les problématiques professionnelles qui en découlent.

\section{Les contenus, de la diffusion à la discussion}

Pour les musées qui les utilisent, les plateformes telles que Facebook, Twitter, Instagram ou Tumblr constituent des supports de diffusion d'informations relatives à l'ensemble de leurs activités. Trois exemples d'usages des RSN par les musées nous permettront d'établir de quelle manière ces dispositifs, qu'on pourrait croire à vocation promotionnelle, relèvent également de la médiation, s'inscrivant dans le prolongement d'actions menées depuis plus de 30 ans.

La Museumweek twittée au musée d'Orsay.

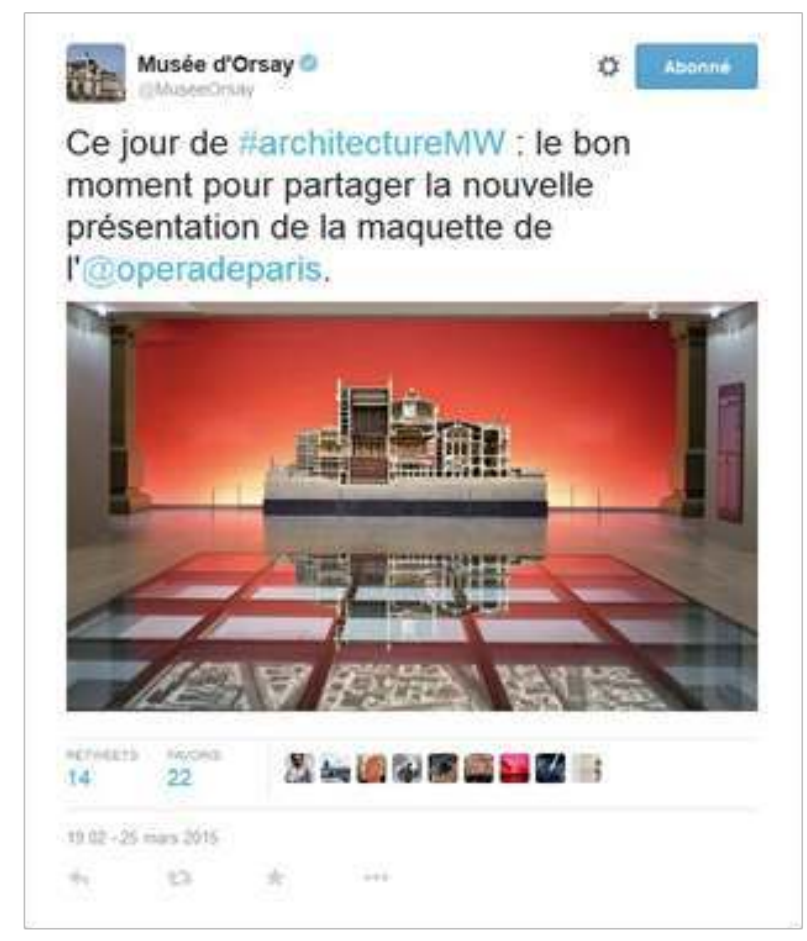

Contrairement aux outils traditionnels de communication asymétrique (communiqués et dossiers de presse, relations avec les médias, campagne d'affichage, brochure de saison...), les RSN permettent d'installer une relation plus équilibrée entre le musée et son public, à partir d'un appareil équipé d'une connexion à Internet. Certains musées s'appuient sur les temporalités du quotidien, comme le château de Versailles qui publie des 
photographies de son jardin sous la neige ou les nombreux musées québécois qui soulignent la Fête nationale le 24 juin. D'autres font référence à la culture populaire : en France, les séries télévisuelles pour les musées d'Angers ou les super-héros pour le musée Saint-Raymond, à Toulouse. Ces publications, si elles peuvent sembler relever d'une communication opportuniste, ancrent les musées dans le quotidien des publics. Elles participent à réduire l'écart avec les visiteurs, favorisant la création et l'entretien d'une relation entre l'institution et ses publics sur laquelle repose toute médiation culturelle, comme le rappellent Serge Chaumier et François Mairesse ${ }^{1}$.

Créé à l'initiative de Twitter, la \#MuseumWeek est un événement qui a lieu en ligne. Plus de 2800 institutions culturelles dans le monde ont participé à la deuxième édition qui s'est tenue du 23 au 29 mars 2015, tweetant chaque jour autour de thématiques communes, telles que les coulisses le lundi, l'architecture le mercredi ou l'offre adressée aux jeunes publics le vendredi. Au-delà de sa fonction promotionnelle, ce dispositif permet de diffuser des informations qui sortent de la programmation et sont peu ou jamais valorisées le reste du temps. Les institutions assurent la médiation de l'établissement lui-même, évoquant son histoire, son architecture ou les spécificités de son organisation. Un tel usage des RSN fait échos aux pratiques de médiation présentielle telle qu'elle est couramment enseignée : la plupart des visites guidées ou des ateliers s'ouvrent par une présentation de l'institution elle-même.

6 En ligne, il est courant d'avoir recours à l'humour. Certains établissements comme le musée de Cluny, à Paris, ont adopté un ton drôle et souvent décalé sur Twitter, jouant de complicité avec leurs abonnés - ce qui n'empêche pas le compte de traiter du Moyen-Âge avec une rigueur toute scientifique. Aller à la rencontre d'éventuels visiteurs en utilisant un ton décalé, voire quelque peu provocateur, s'inscrit dans le prolongement des pratiques classiques de la médiation, comme l'indiquent Claire Merleau-Ponty et JeanJacques Ezrati : «[le public] espère découvrir et apprendre en même temps qu'il pense se divertir. Il attend que soit stimulée sa curiosité, provoquée son admiration, enrichies ses connaissances et renforcées ou ébranlées ses convictions $»^{2}$.

7 La médiation culturelle se construit dans un échange entre les publics et l'institution, qui ne peut faire fi de l'expérience des visiteurs, là où la communication institutionnelle tend vers la diffusion d'un message descendant, adressé à un public captif. Les actions que nous venons de lister permettent aux musées d'installer, de développer et d'entretenir des relations de proximité, de confiance et de complicité avec leurs publics en ligne. Loin de supplanter les outils « traditionnels » de médiation (cartels et textes de salle, dépliants et guides de visite, ateliers, visites guidées et contées...), les RSN permettent la mise en place de nouvelles dynamiques entre les institutions et leurs publics.

\section{Les publics, entre appropriation et participation}

L'usage des RSN par les musées réactive la promesse de l'engagement des publics dans la construction du propos de l'institution, formulée dès les années 1970 par Georges-Henri Rivière et Hugues de Varine à travers le projet des écomusées ${ }^{3}$, puis par le mouvement de la Nouvelle muséologie. L'appartenance à un territoire donné fait de ses habitants une communauté, que l'écomusée souhaite associer à tous les niveaux : création, animation et administration. Or, en ligne, ce qui fait communauté pour les fans d'un musée, c'est leur intérêt pour ses collections et ses thématiques, plus que leur appartenance à un territoire. Pourtant, les RSN ne constituent-ils pas un territoire numérique? Depuis la popu- 
larisation du Web dans les années 1990, le vocabulaire qui l'accompagne est caractérisé par d'abondantes références à la navigation («surfer », «navigateur », "visites »...), accentuant l'idée qu'il s'agit d'une terre inconnue à explorer. Si Internet promet de toucher les publics en ligne partout dans le monde, l'expérience montre une réalité plus subtile. À Nouméa, le musée de Nouvelle-Calédonie utilise, avec succès, la fonctionnalité "événement» de Facebook pour informer les visiteurs de la tenue d'ateliers et de spectacles, tout autant que pour prolonger ces activités en ligne. Or, avec $47 \%$ des habitants de l'archipel inscrits sur cette plateforme ${ }^{4}$, les quelque 2200 fans de la page ${ }^{5}$ illustrent la pertinence, pour une institution culturelle, d'une stratégie numérique tenant compte des spécificités de son public local. Avec un nombre d'abonnés sur Facebook proche, la situation est pourtant tout autre au musée Jeanne d'Albret ${ }^{6}$, à Orthez, petit musée associatif consacré à l'histoire du protestantisme en Béarn. Majoritairement localisés à l'extérieur de la région Aquitaine, les 2500 fans Facebook ${ }^{7}$ sont le résultat d'une stratégie en ligne visant à développer le rayonnement du musée à l'extérieur de son territoire local.

Informer les visiteurs par Facebook au musée de Nouvelle-Calédonie.

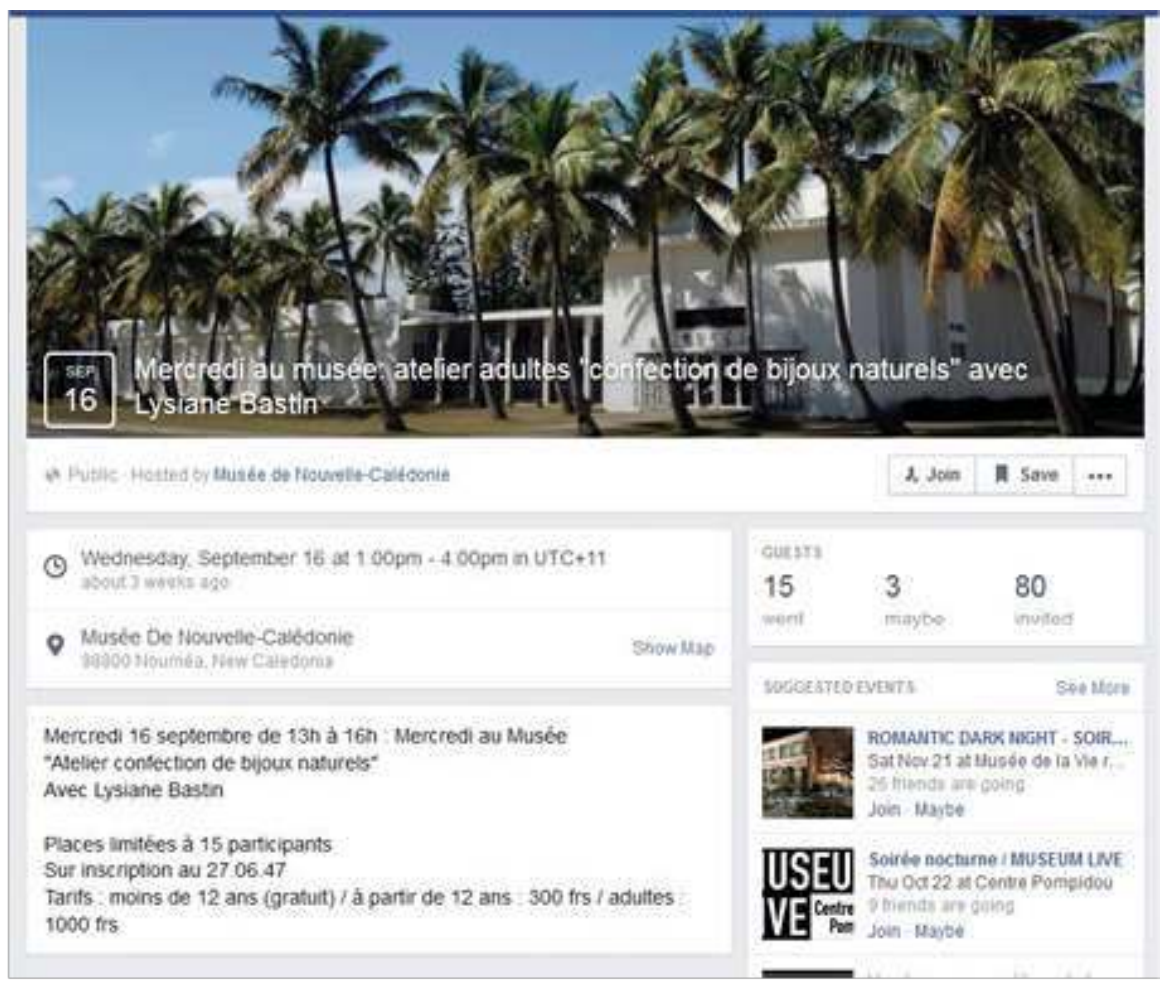

En utilisant les plateformes sociales, les publics deviennent actifs dans la diffusion et la publication des contenus qu'ils produisent autour de leur visite. En postant sur les RSN les photographies qu'ils prennent ou en écrivant des articles sur leurs blogs, ces visiteurs peuvent même développer des compétences éditoriales et créatives. Autrefois, les commentaires qui naissaient lors d'une visite dépassaient rarement le cadre familial et/ ou amical. Aujourd'hui, les musées ont la possibilité de prendre connaissance de ces réactions et de ces productions venant des publics, d'y réagir et/ou de les valoriser s'ils le souhaitent. Au musée du quai Branly, nous avons mis en place un dispositif de médiation associant l'un de nos abonnés les plus investis sur Twitter, Laurent Granier8. 
Collectionneur d'art d'Afrique et d'Océanie sans être professionnel du monde scientifique ou culturel, il a entrepris d'explorer les bases de données en ligne du musée, publiant ses coups de cœur sur son compte Twitter. À l'automne 2014, à l'occasion de l'exposition L'Éclat des Ombres, L'Art en noir et blanc des îles Salomon, Laurent Granier a publié une série de tweets lors de la semaine d'ouverture, fruit d'une sélection de pièces organisée autour de thématiques qu'il a lui-même définies. En amont, il avait rencontré la commissaire de l'exposition pour évoquer son projet. Ses publications ont enrichi le propos de l'exposition, approfondissant certains points qui n'y étaient pas traités.

Si ce type de dispositifs tend à créer de nouvelles hiérarchies entre visiteurs investis et publics moins réactifs, il démontre néanmoins la possibilité pour des personnes qui ne sont pas "qualifiées" (par des études, un diplôme ou une expérience professionnelle dans le secteur culturel) d'être associées à la production du discours entourant une exposition et ce, sur la base d'un savoir développé par curiosité ou par passion. Celui-ci ne se substitue aucunement au discours scientifique, qui se déploie notamment dans l'espace d'exposition et sur bien d'autres supports, mais il vient le prolonger en ligne, auprès des utilisateurs des RSN abonnés aux pages du musée.

\section{L'essor du community management}

11 Né avec les prémices du Web social (les groupes de discussion en ligne, les forums), le community management a pour objectif d'animer une communauté réunie autour d'un centre d'intérêt ou d'une passion. Or, comme le rappellent Sarah Barrett et Olivier Richard, «si la médiation est une fonction, il n'est pas évident qu'elle s'incarne dans un métier unique $»^{9}$. Avec le développement des outils numériques, le community management tend à devenir un de ces métiers, à la fois partie intégrante de l'institution culturelle et en prise directe avec les publics. L'agent en charge du community management diffuse les réactions et les commentaires des visiteurs sur la programmation et les collections du musée, relayant cette parole auprès des instances de décision de l'établissement ${ }^{10}$. 
Un système de médiation via Twitter, conçu et animé par un collectionneur, au musée du quai Branly

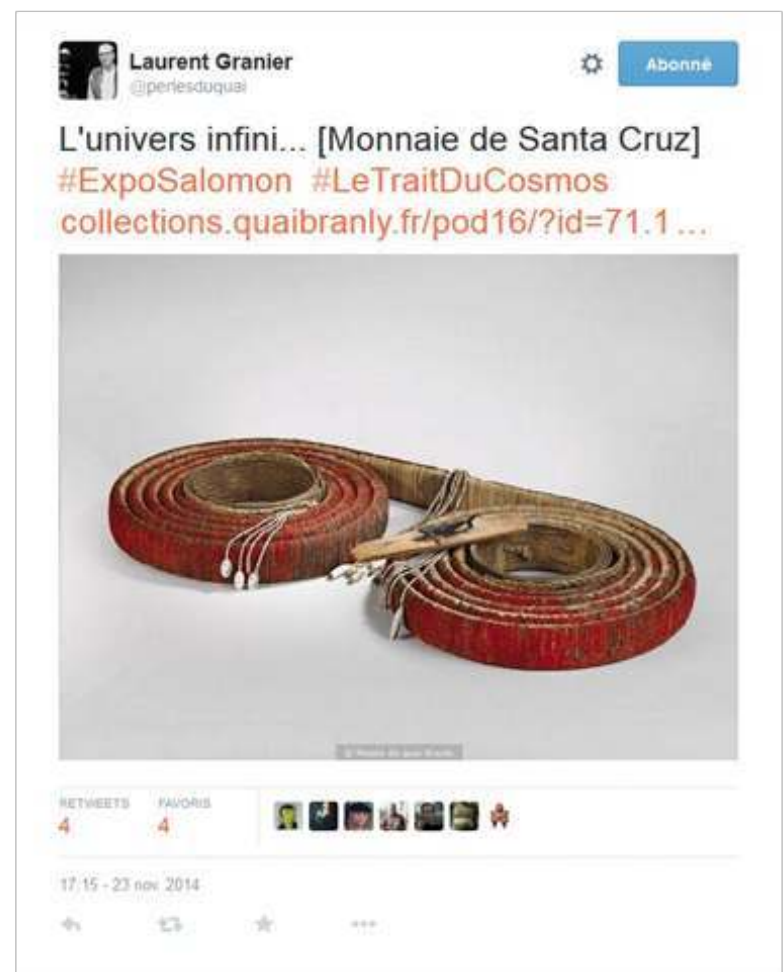

$12 \mathrm{Au}$ quotidien, le community management est pratiqué par trois types d'agents: les community managers dont c'est l'une des tâches (exceptionnellement l'unique); des médiateurs/trices qui, du fait de leurs fonctions en relation avec les visiteurs sont amenés à intervenir sur les RSN de leur institution; d'autres agents, dont les fonctions ne sont pas toujours reliées aux publics et qui n'ont pas nécessairement accès aux comptes «officiels» mais qui, par leurs actions sur leurs comptes personnels, participent à l'animation de la communauté des fans du musée, parfois aux plus hauts niveaux de la hiérarchie $^{11}$. En France, l'exemple des musées d'Angers ${ }^{12}$ est particulièrement parlant. L'animation des comptes Facebook et Twitter, ouverts respectivement depuis 2010 et 2011, est assurée par une équipe de quatre personnes : une chargée de communication, deux agents du service des publics (une médiatrice et la directrice) et une chargée de récolement. Cette diversité de profils permet d'aborder plusieurs thématiques tout en s'exprimant avec la même voix : tour à tour, les comptes publient informations pratiques, coulisses de montage d'exposition, œuvres sorties des réserves. Plusieurs actions de médiation in situ sont fidèlement adaptées en ligne, en profitant de fonctionnalités propres aux plateformes, avec un ton léger et décalé qui s'inscrit dans les usages de Twitter.

13 Lorsqu'il est formulé comme une mission en tant que telle, le community management est souvent attribué à des agents en charge du site web et/ou des outils traditionnels de communication, assurant une certaine cohérence éditoriale. À l'heure où le nombre d'abonnés ne cesse de croître et où les publics ont pris l'habitude de pouvoir les interpeller en ligne, la reconnaissance de la pertinence de leur travail par leurs hiérarchies constitue un enjeu majeur pour beaucoup d'agents assurant le community management. La création du groupe de travail $\# \mathrm{CMmin}^{13}$ en 2013 , qui réunit les 
community managers des établissements relevant du ministère de la Culture et de la Communication, constitue une reconnaissance importante car venant de la tutelle.

Toute médiation n'est pas obligatoirement reliée aux RSN et, réciproquement, les RSN ne servent pas qu'à faire de la médiation. Néanmoins, accepter d'entendre les commentaires des publics en ligne, même s'ils ne cadrent pas avec la communication institutionnelle, offre l'opportunité pour les musées d'installer avec ces visiteurs une collaboration dans l'élaboration du discours entourant les œuvres et la programmation. Une telle dynamique s'inscrit dans le prolongement des missions de médiation et diffusion du savoir qui sont celles des musées. L'enjeu, éminemment politique, c'est l'encapacitation (en anglais, empowerment) qui passe par l'amélioration de la circulation de l'information, la valorisation de l'investissement des visiteurs et de leurs productions, l'association des publics à la prise de décision.

Bien que les visiteurs se heurtent aux limites de ces plateformes - fermeture des écosystèmes, enjeux de propriété intellectuelle, contingences techniques... -, les RSN peuvent être des outils parmi d'autres au service d'une politique d'encapacitation des publics : une authentique co-construction du propos et des contenus, dans laquelle le musée continue de fournir son expertise scientifique sur les collections (documentation, information, exposition, médiation) et les publics peuvent apporter leurs positionnements, leurs interrogations, leurs créations. Mais cet objectif nécessite d'accepter que les RSN puissent être des outils de médiation, en ne les limitant pas à un usage promotionnel.

\section{NOTES}

1. Chaumier, S. et Mairesse, F. La médiation culturelle. Paris : Armand Colin, 2013, p. 34.

2. Merleau-Ponty, C. et Ezrati J.-J. L'exposition, théorie et pratique. Paris : L'Harmattan, 2005.

3. À ce propos, voir La Muséologie selon Georges-Henri Rivière, collectif, Dunod (Bordas), 1989, et notamment De Varine, H. La participation de la population. Principes, pp. 312-315.

4. Observatoire numérique de Nouvelle-Calédonie, Faits et chiffres : internet fixe en NouvelleCalédonie, janvier 2014. : www.observatoire-numerique.nc/sites/default/files/pdf/actualites/ internet_et_telephonie_fixe_-_mars_2014.pdf.

5. Chiffre constaté en juillet 2015, sur www.facebook.com/pages/Mus\%C3\%A9e-de-Nouvelle-Cal\% C3\%A9donie/173997297476.

6. Abadie-Laborde, C. Musée Jeanne d'Albret, intervention aux Rencontres numériques le 7 octobre 2014 : www.rencontres-numeriques.org/2014/mediation/? action=restitution.

7. Chiffre constaté en juillet 2015, sur www.facebook.com/museejeannedalbret.

8. https://twitter.com/perlesduquai.

9. Richard, O. et Barrett S. Les Médiateurs scientifiques en Europe : une diversité de pratique ; une communauté de besoin, La Lettre de l'OCIM, n¹35, mai-juin 2012, pp. 5-12, cité dans Chaumier, S. et Mairesse, F. La médiation culturelle. Paris : Armand Colin, 2013.

10. À propos de l'ouverture de la médiation culturelle aux outils numériques, voir Chaumier, S. et Mairesse, F. La médiation culturelle. Paris : Armand Colin, 2013, pp. 135 et 138-140. 
11. Comme l'illustre sur Twitter l'activité d'Oliver Gabet, directeur général des Arts Décoratifs, de Philippe Bélaval, président du Centre des Monuments nationaux ou encore de Catherine Pégard, présidente du Château de Versailles.

12. Guillemant, J. Musées d'Angers, intervention aux Rencontres numériques le 7 octobre 2014 : www.rencontres-numeriques.org/2014/mediation/? action=restitution.

13. http://cmmin.fr/

\title{
RÉSUMÉS
}

Analysant les enjeux et les perspectives pour les musées francophones de l'utilisation des plateformes socio-numériques, l'auteur montre comment ces outils de médiation permettent d'ancrer les musées dans le quotidien des publics, d'entretenir des relations de proximité, voire de complicité entre l'institution muséale et ses visiteurs et met en évidence le rôle stratégique joué en la matière par le community manager.

\section{INDEX}

Mots-clés : résaux sociaux, médiation culturelle, musée

\author{
AUTEUR \\ SÉBASTIEN MAGRO \\ chargé de projets nouveaux médias, Service du développement numérique, Direction du \\ développement culturel du musée du quai Branly \\ sebastien.magro@gmail.com
}

